\title{
The efficacy of a preparatory phase of a touch-based approach in treating chronic low back pain: a randomized controlled trial
}

This article was published in the following Dove Press journal:

Journal of Pain Research

20 April 2017

Number of times this article has been viewed

\author{
Federico Zangrando' \\ Giulia Piccinini² \\ Clara Tagliolini' \\ Gabriella Marsilli \\ Marco losa ${ }^{3}$ \\ Maria Chiara Vulpiani ${ }^{2}$ \\ Teresa Paolucci' \\ 'Complex Unit of Physical Medicine \\ and Rehabilitation, Policlinico \\ Umberto I Hospital, "Sapienza" \\ University of Rome, Rome, Italy; \\ ${ }^{2}$ Unit of Physical Medicine and \\ Rehabilitation, Sant'Andrea Hospital, \\ "Sapienza" University of Rome, \\ Rome, Italy; ${ }^{3}$ Clinical Laboratory of \\ Experimental Neurorehabilitation, \\ Santa Lucia Foundation, Rome, Italy
}

Correspondence: Teresa Paolucci Complex Unit of Physical Medicine and Rehabilitation, Policlinico Umberto I Hospital, "Sapienza" University of Rome, Piazzale Aldo Moro 5, 00185 Rome, Italy Tel +393479338625

Fax +39064 991 4552

Email teresapaolucci@hotmail.com
Background: Massage therapy is an important element of rehabilitation in the treatment of chronic low back pain (CLBP). The objective of this study was to determine the relative efficacy of massage therapy between traditional massage and a new massage approach for CLBP. We also examined whether any reduction in pain was linked to interoceptive awareness and parasympathetic activation.

Methods: A single-blind, randomized, controlled trial of 51 patients who were allocated into a traditional massage therapy group (TMG; $\mathrm{N}=24$, mean age: $50.54 \pm 9.13$ years) or experimental massage therapy group ( $\mathrm{SMG} ; \mathrm{N}=27$, mean age: $50.77 \pm 6.80$ years). The primary outcome was the reduction in pain per the visual analog scale (VAS); the secondary outcome measures were multidimensional pain intensity on the McGill Pain Questionnaire, pain-related disability per the Waddel Disability Index, interoceptive awareness per the Multidimensional Assessment of Interoceptive Awareness Questionnaire, quality of life per the Short Form - 12 Health Survey, and heart rate variability, expressed as the coherence ratio (CR) by photoplethysmography. The following outcome measures were assessed at baseline, at the end of the treatment program, and at the 3-month follow-up. The mean and standard deviation were calculated for continuous data. Mann-Whitney $U$ test was used to perform between-group comparisons, Friedman's analysis was used for data on the 3 assessment times in each group, and Spearman's $R$ coefficient was used to analyze correlations.

Results: Both approaches had a positive result on pain, an effect that was more acute in the SMG versus TMG for all pain scales, with better maintenance at the 3 -month follow-up (VAS $p=0.005$ and $p=0.098$; Waddell Index $p=0.034$ and 0.044; McGill total $p=0.000$ and 0.003). In the SMG, CR scores were significant at baseline and at the end of the treatment program ( $p=0.000$ and 0.002 ).

Conclusion: The new massage approach with a preparatory phase that is pleasant to the touch was more effective than the traditional approach for CLBP.

Keywords: perception, rehabilitation, quality of life, pain memory, massage

\section{Introduction}

CLBP is one of the most common musculoskeletal problems in modern society. Between $70 \%$ and $80 \%$ of adults experience it at least once in their lives. ${ }^{1}$ The management of CLBP comprises a range of strategies, including surgery, drug therapy, and rehabilitative interventions, such as massage therapy. ${ }^{2}$ Massage therapy has the potential to minimize CLBP and accelerate the return to normal function. ${ }^{3}$ The Ottawa Panel demonstrated that massage interventions affect short-term improvements in subacute and CLBP symptoms, decrease disability immediately after treatment, and provide short-term relief when combined with therapeutic exercise and education. ${ }^{4}$ 
No clinically meaningful difference between relaxation and structural massage has been observed with regard to relieving disability or symptoms in CLBP. ${ }^{5-7}$

The benefits of massage therapy are enhanced when it is accompanied by routine physical therapy - particularly rehabilitative exercises - indicating that massage is an effective treatment for CLBP versus placebo and other active treatment options (such as relaxation), especially in the short term. ${ }^{8-10}$ A significant aspect of massage in CLBP is its ability to relax the patient, which has been linked to HRV. Specifically, short-term relaxation therapy improves autonomic balance and promotes cardiovascular health, decreasing HRV, correlating with blood pressure. ${ }^{11,12} \mathrm{HRV}$ is often applied as an index of balance in the ANS; as a relaxation therapy, massage can improve autonomic balance and improve cardiovascular health by establishing a sympathovagal equilibrium, which can help a patient shape his perception of bodily signals. ${ }^{13-15}$

In CLBP, as in other chronic illnesses, fluctuations in physical symptoms and emotional states correlate with IA. Moreover, IA, which is one's sense of the physiological condition of his body, might have significant function in mediating self-rated health, particularly in the perception of chronic pain. ${ }^{16-19}$ Poor access to bodily signals restricts a patient's ability to integrate them during emotional processing, which, by extension, precludes optimal emotional SR. ${ }^{20}$ In CLBP, the patient experiences reweighting of proprioceptive information, altered sensitivity to exteroceptive stimuli, and disrupted IA of the state of the body. ${ }^{21}$ Also, pain-related changes in cortical areas that are allocated to pain sensation appear to experience stress, which could elicit pain memory. ${ }^{22}$

Together, pain experiences and pain memory affect the maintenance of chronic pain. ${ }^{23}$ People with chronic pain have difficulty directing their attention away from it; to this end, a mental strategy that incorporates focused attention and distraction with non-painful stimuli or a self-generated sub-nociceptive image positively modulates the perception of pain intensity. ${ }^{24}$ Certain massage techniques, such as RMB, use touch and words to enhance body awareness of physical sensations and emotional states. ${ }^{25}$

Based on these findings, the objective of our study was to determine the relative efficacy of a new massage therapy in reducing pain in CLBP as the primary outcome. Also, considering the lack of studies that have correlated CLBP with IA and HRV, our secondary purpose was to examine whether and how the decrease in pain is linked to IA and HRV by measuring the $\mathrm{CR}$, which reflects the average stress that is experienced by patients. We hypothesized that a preparatory phase that is pleasant to the touch, before every session of the traditional massage treatment, directs the attention of the patient away from his pain, increasing the efficacy of massage in reducing pain.

\section{Ethical approval and consent to participate}

All participants signed informed consent forms after receiving detailed information on the study's aims and procedures, as per the Declaration of Helsinki. Written consent for the use of the photographs in this study was obtained. This study was approved as a clinical trial (ClinicalTrials.gov identifier NCT02646280) and by the ethical committee of "Sapienza" University of Rome (registration number 3791/15).

\section{Methods}

This study was a single-blind, randomized, controlled trial that took place from July 2015 to January 2016. Patients were recruited from the outpatient rehabilitation clinic of Policlinico Umberto I Hospital, Rome. The inclusion criteria were patients aged between 30 and 60 years with a diagnosis of chronic nonspecific LBP for at least 3 months and VAS $\geq 3$ in the last week. The exclusion criteria were acute LBP; LBP due to specific causes; concomitant rheumatic, neurological, or oncological disease; previous back surgery; severe cognitive impairments; and pregnancy.

Eligible patients were referred to a physiatrist who provided them with detailed information on the experimental protocol. A standardized, blinded assessment at baseline and at the follow-up was performed by the same examiner to minimize any potential bias when performing the clinical examination and recording the data. The examiner did not have access to the clinical or radiological examination results, maintaining the blinding and limiting the risk of biased observations.

Fifty-eight patients were screened, 51 of whom were enrolled and then randomized to the $\mathrm{TMG}(\mathrm{N}=24$, mean age: $50.54 \pm 9.13$ years $)$ or SMG $(\mathrm{N}=27$, mean age: $50.77 \pm 6.80$ years) at a 1:1 ratio, according to a computer-generated randomization list (Figure 1) using SPSS 10.1 (SPSS Inc., Chicago, IL, USA) through a random selection of $50 \%$ of cases. The allocation was concealed from the patients and examiner; patients were allocated according to a printed computer-generated list, and each number was covered with a patch by a researcher who was not involved in the patients' assessments. The patches were removed successively after the inclusion of each patient into the study by another researcher who did not participate in the patient assessments, revealing the allocation. Conversely, the clinical assessor was unaware of the group allocation. 


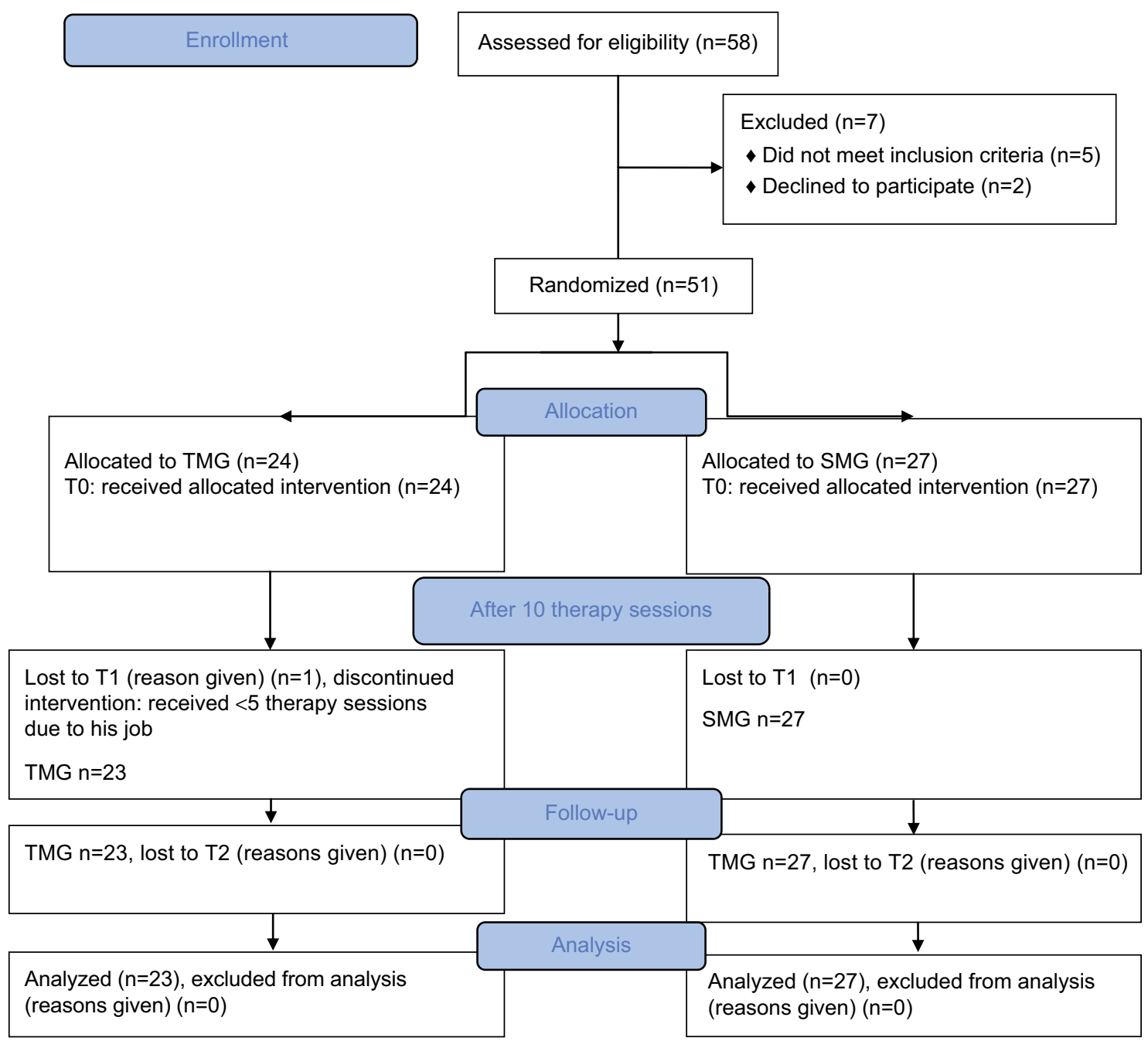

Figure I Flow chart of the study.

Abbreviations: TMG, traditional massage therapy group; SMG, experimental massage therapy group; T0, baseline; TI, end of the treatment program; T2, 3-month follow-up.

Sociodemographic and clinical data were collected at baseline. The following outcome measures were assessed at baseline (T0), at the end of the treatment program (T1), and at the 3-month follow-up (T2). HRV, based on CR, was measured only during the initial contact and at the last treatment session to detect any change in values after treatment and the $\mathrm{T} 2$.

Pain intensity was the primary outcome and was measured using the VAS. ${ }^{26}$ The secondary outcome measures were multidimensional pain intensity per the MPQ ${ }^{27}$ pain-related disability per the Waddel Disability Index, ${ }^{28}$ IA per the MAIA, ${ }^{29}$ quality of life per the SF-12,30,31 and HRV expressed as CR using a photoplethysmograph (emWave software Heartmath HMA 6020). ${ }^{32}$

The VAS is a psychometric tool that evaluates pain intensity from 0 to $10(0=$ absence of pain and $10=$ severe pain $)$. The patient expresses his pain intensity by indicating the point along a continuous line from 0 to $10 \mathrm{~cm}$. The MPQ is a mul- tidimensional scale that assesses clinical pain. It allows one to evaluate the sensory, affective, and emotional clinical condition of the patient regarding his pain. The questions are divided into 11 sensitive categories and 4 affective categories. The patient is asked to give a score from 0 to 3 for each category. The result of the test consists of 7 scores: PRIS somatosensory score (0-35.5), PRIA affective score (0-21.3), PRIE emotional score (0-4.60), Mixed Pain Rating Index score mixed (0-16.1), NWC (0-20), PPI (0-5), and S/A. We considered the total score (McGill Tot) to range from 0 to 78 . The MPQ has been translated into many languages, including Italian.

The Waddel Disability Index is used to evaluate disability in CLBP regarding daily living activities. The questions, divided into 9 items, are answered with a yes or no response, with total scores ranging from 0 to 9 ( $>5$ indicates significant disability). This index examines a patient's autonomy with respect to lifting, sitting, standing, traveling, walking, sleeping, social life, sex life, and putting on footwear. 
The MAIA multidimensional scale was used to assess IA and consists of 32 items, clustered into 8 subscales with a range of 0 to 5 (= greatest level of awareness) for each: N, ND, NW, AR, EA, SR, BL, and T.

The SF-12 is a multipurpose, short-form survey that comprises 12 questions, all of which are selected from the SF-36 Health Survey. The questions are combined to create 2 scales that determine physical and MH. The PCS is represented by 4 domains: physical function, PR, BP, and GH. The MCS is composed of MH, SF, RE, and VT. Physical and $\mathrm{MH}$ composite scores are computed using the scores on the 12 questions and range from 0 to 100 (worst and best health state, respectively).

HRV is a measurement of cardiac function and reflects heart-brain interactions and the dynamics of the ANS. A photoplethysmograph records HRV, which is usually influenced by various levels of stress, ${ }^{33}$ and was used to assess patient compliance and behavior during contact (ie, when the physical therapist touched the patient's back for the first time). Through software that analyzes heart signal patterns, we obtained a parameter, termed the $\mathrm{CR}$, which detects the average stress that is experienced by patients. High CR values indicate greater coherence in the heart signal pattern and, consequently, a rise in HRV, which demonstrates the prevalence of the parasympathetic system (reduction in stress levels); in contrast, low values reflect decreased coherence in heart signal patterns and thus a declining HRV, which signifies the predominance of the sympathetic system (increase in stress levels).

All patients were instructed not to take any medications for low back pain (VAS <5) (eg, NSAIDs, muscle relaxants, antidepressants, and corticosteroids) during the study protocol and not to undergo other rehabilitation approaches (those who did so were dropped from the study). During the rehabilitation sessions, no patients reported any increase in pain. We excluded patients who attended fewer than 9 sessions.

All enrollees were subjected to contact before massage treatment to measure the basal CR, detected by photoplethysmography of a patient's earlobe: with patient in the prone position, the examiner placed the palm of his hand on sore and painless lumbar regions ( 6 touches, 3 on each side). The examiner was blinded to the patient allocation.

We proposed 10 intervention rehabilitative sessions for each rehabilitation group, each lasting $30 \mathrm{~min}$ and performed 3 times per week.

The number of sessions and the time of each session were chosen, considering routine good clinical medical practice with respect to massage therapy for $\mathrm{CLBP}^{8}$ and the SIMFER guidelines (http://www.simferweb.net).

\section{Traditional rehabilitative massage program}

We applied the following massage techniques ${ }^{34}$ to the lumbar region of patients in both groups (TMG and SMG) (Figure 2):

- touch surface (10 min): both hands held open with the fingers gliding slowly over the skin without pressure or direction.

- Deep touch (5 min): both hands held open with the fingers gliding slowly over the skin, with increased pressure in the direction of the muscle bundles. "Touching" is the maneuver that begins and ends a massage session, consisting of slow and light movements with the surface of the palm making complete contact with the surface of the body. The PT must maintain balance on his feet and should not apply weight to the wrists during the massage.

- Static pressure (5 min): with fists or the palms, compressions are performed perpendicularly to the surface of the muscle above the iliac wings, on the spinous processes, and on the vertebral facets.

- Dynamic pressure (5 min): this technique is similar to static pressure, with the addition of slight friction against the surface of the skin.

- Kneading (5 min): pinching and rolling (pince roulée) - a maneuver of detachment that affects the skin. The skin is lifted between the thumb and forefinger of both hands together or one hand for small areas, "walking" the index, middle, and ring fingers, as if testing the density of a soft substance.

\section{New rehabilitative massage program}

Using the same massage techniques and durations, we added various elements:

- a preparatory phase that was pleasant to the touch: at the beginning of each massage session, the patient was asked to sense the pleasant "contact" (comfortable warmth) of the therapist's hands (slight pressure with the palm to the region of the back without pain). Then, the patient had to memorize the sensation of the pleasant touch and

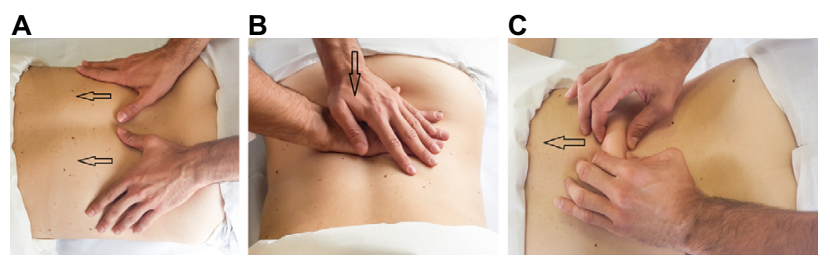

Figure 2 Traditional rehabilitative massage program.

Notes: Progression of the different phases: surface and deep touch $(\mathbf{A})$, static and dynamic pressure (B), pinching and rolling $(\mathbf{C})$. 
relaxation. Afterward, the PT asked the patient to "bind" the pleasant and pain-free sensation to the painful area. At this point, the PT touched the painful area of the back (Figure 3 ). The session continued with traditional massage.

- Language as therapy: to assess the patient's ability to recount and describe what he felt during the massage. For example, in the initial treatment session, a patient might have been unable to perceive the sensation of heat throughout the painful lower back, perceiving it to be as "impenetrable as cement". As the acceptance of and confidence in the massage and PT increase, the same patient reports his back as becoming "adaptable and as permeable as sand", becoming able to feel the heat throughout his body. During the massage session, the patient had an active role, providing continuous feedback through dialog with the PT. The patient was actively involved in the treatment with regard to emotional and cognitive perception.

\section{The setting}

The massage was performed by 2 PTs who had been trained in the massage treatment techniques for at least 3 years. The PTs alternated between treatment groups at a ratio of 1:1 (1 TMG, then $1 \mathrm{SMG}$ ) to avoid operator-dependent bias. The session was performed in a comfortable and quiet environment, with access to natural light, only in the presence of the PT and the patient. In the TMG, the patient was asked during the session to relax and communicate with the PT only if there were unpleasant sensations during the treatment.

\section{Sample size calculation}

Assuming an average reduction in pain of $\sim 2$ points in the experimental group $(\mathrm{SMG})$ and $\sim 1$ point in the control

A

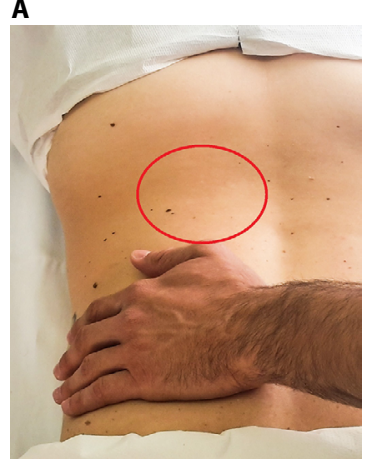

Figure 3 Preparatory phase to the massage.

Notes: (A) The pleasant "contact" in the area of the back without pain. (B) The physiotherapist touches the painful area on the back. group, as measured on the VAS, and a standard deviation of 1.5 and 0.75 , respectively (obtained assuming a coefficient of variation of $75 \%$ of average reductions), we calculated a requirement of 17 patients per group (PASS Software ${ }^{\odot}$ ) by Student's $t$-test, with a power of $80 \%$ and a 0.05 alpha error. With an estimated dropout rate of $10 \%$, the minimum number of patients per group was 19 . However, our cohort was larger (23 versus 27), because we wanted to respect the time limits of the enrollment per the ethics committee (from July 2015 to October 2015) and because we decided to enroll all patients who sought treatment at our clinic to avoid creating a wait list of untreated patients.

\section{Statistical analysis}

The mean and standard deviation were calculated for continuous data. Because clinical scale scores are ordinal measures, they were summarized using median and quartiles and are reported using box and whisker plots. Betweengroup comparisons were performed by Mann-Whitney $U$ test, whereas within-group comparisons were made using Friedman's analysis for data on the 3 assessment times in each group. All analyses were performed using SPSS v15. Spearman's $R$ coefficient was computed to analyze correlations. The critical alpha level was set to 0.05 for all analyses. Our approach also considered the minimal clinically important difference for VAS scores. ${ }^{35}$ Patients who abandoned the protocol or refused to be retested at $\mathrm{T} 1$ or $\mathrm{T} 2$ were considered to have dropped out. Finally, we compared the number of subjects who maintained a meaningful clinical change in pain $(30 \%),{ }^{36}$ quantified as a 1.5 cm shift on the VAS: for these analyses, the odds ratio (and the relevant $95 \%$ confidence interval) was computed by chi squared analysis, for which the $p$-value was considered to be statistically significant if it was $<0.025$, because this was a secondary-level analysis.

\section{Results}

Of the 58 patients who were screened, 51 were enrolled and randomized into 2 groups: 24 in the TMG and 27 in the SMG. In the TMG, 1 patient dropped out for job-related issues and did not complete the therapy sessions ( $<5$ sessions) or tests (Figure 1); thus, the statistical calculation was performed for a sample size of 23 patients in the TMG and 27 patients in the SMG. There were no significant differences in age, gender, or BMI at the initial assessment: the demographic and clinical characteristics are shown in Table 1 ( $p>0.05$ for all parameters). In addition, there were no statistically significant or clinically meaningful differences at baseline in terms of 
Table I Demographic and clinical data of participants at baseline

\begin{tabular}{|c|c|c|}
\hline $\begin{array}{l}\text { Characteristics of the } \\
\text { sample }\end{array}$ & TMG & SMG \\
\hline Number of participants & 24 & 27 \\
\hline Age (years) & $50.54 \pm 9.13$ & $50.77 \pm 6.80$ \\
\hline \multicolumn{3}{|l|}{ Gender } \\
\hline Female & $14(58.33)$ & $15(55.56)$ \\
\hline Male & $10(41.67)$ & $12(44.44)$ \\
\hline Mass (kg) & $72.50 \pm 13.15$ & $70.07 \pm 11.88$ \\
\hline Stature $(\mathrm{m})$ & $1.70 \pm 0.09$ & $1.69 \pm 0.08$ \\
\hline Body Mass Index $\left(\mathrm{kg} / \mathrm{m}^{2}\right)$ & $24.72 \pm 2.55$ & $24.30 \pm 2.68$ \\
\hline Primary school & $0(0)$ & $0(0.44)$ \\
\hline Secondary School & $2(8.33)$ & $3(11.11)$ \\
\hline High School & $10(41.67)$ & $7(25.93)$ \\
\hline University & $12(50)$ & $17(62.96)$ \\
\hline Employed & $16(66.67)$ & $19(70.38)$ \\
\hline Retired & $2(8.33)$ & $0(0)$ \\
\hline Unemployed & $2(8.33)$ & $2(7.4)$ \\
\hline Never worked (Housewife) & $4(16.67)$ & $6(22.22)$ \\
\hline Married & $12(50)$ & $14(51.85)$ \\
\hline Single & $12(50)$ & $13(48.15)$ \\
\hline
\end{tabular}

Note: Data shown as mean \pm standard deviation or $\mathrm{n}(\%)$.

Abbreviations: TMG, traditional massage therapy group; SMG, experimental massage therapy group.

clinical scale scores between groups at baseline $(p>0.05$, Mann-Whitney $U$ test). There were significant changes in the novel versus control group for most parameters at the end of the treatment by Friedman's analysis (Table 2): in the SMG, $p<0.001$ for all parameters, whereas CR and SF-12 scores in the TMG were not significant.

By Mann-Whitney $U$ test, the SMG had better results: the CR was significant at $\mathrm{T} 1$ and $\mathrm{T} 2(p=0.000$ and 0.002$)$, and VAS and McGill PPI scores were significant only at T1
( $p=0.005$ and 0.013) (Figure 4); further, the McGill Tot (Figure 5) and PRIA scores were significant at T1 and T2 ( $p=0.000$ and 0.003 and $p=0.001$ and 0.002 , respectively), as was the Waddel Index ( $p=0.034$ and 0.044). Differences in McGill PRIS and PRIE scores were significant at $\mathrm{T} 1(p=0.005$ and $0.025)$. MAIA scale scores differed significantly only at $\mathrm{T} 1$ for MAIA-N, MAIA-AR, and MAIA-Tot ( $p=0.045,0.032$, and 0.023 respectively). There were no significant differences in SF-12 scores between groups. Spearman's $R$ coefficient between VAS and CR was $R=-0.289(p=0.042)$ at T2 and $R=-0.516(p<0.001)$ at $\mathrm{T} 1$.

Finally, we compared the number of subjects who obtained a minimum clinically important change in VAS score $(1.5 \mathrm{~cm})$ that was maintained at $\mathrm{T} 2$. The odds ratio was 7.5 (95\% confidence interval: $1.43-39.47)$, which was statistically significant (chi squared $=6.802, p=0.0091$ ).

\section{Discussion}

The results of our study are encouraging with regard to our hypothesis that a preparatory phase that is pleasant to the touch directs the attention of the patient away from pain; in combination with conventional techniques, this approach increases the efficacy of traditional massage in reducing chronic pain: the experimental treatment had an effect compared to the traditional treatment. Both approaches in the TMG and SMG mitigated pain in CLBP, but in the SMG, this improvement was much more acute compared with the TMG on all pain scales, with better maintenance at 3 months follow-up (Waddell Index, McGill PRIA, Tot, and NWC, as shown in Table 2).

Table 2 Data and statistical analyses

\begin{tabular}{|c|c|c|c|c|c|c|c|c|c|c|c|}
\hline \multirow[t]{2}{*}{ Scales } & \multicolumn{3}{|l|}{ TMG } & \multirow{2}{*}{$\begin{array}{l}\text { Friedman } \\
\text { analysis } \\
p \text {-value }\end{array}$} & \multicolumn{3}{|l|}{ SMG } & \multirow{2}{*}{$\begin{array}{l}\text { Friedman } \\
\text { analysis } \\
p \text {-value }\end{array}$} & \multicolumn{3}{|c|}{$\begin{array}{l}\text { TMG versus SMG } \\
\text { p-values }\end{array}$} \\
\hline & TO & TI & T2 & & TO & TI & T2 & & TO & TI & T2 \\
\hline $\begin{array}{l}\text { Coherence } \\
\text { ratio }\end{array}$ & $0.84 \pm 0.33$ & $0.89 \pm 0.29$ & $0.86 \pm 0.29$ & 0.274 & $0.9 \pm 0.27$ & $1.26 \pm 0.32$ & I.I $13 \pm 0.32$ & $<0.001$ & 0.393 & $<0.001$ & 0.003 \\
\hline HRV & $67.70 \pm 5.5 \mathrm{I}$ & $66.91 \pm 4.91$ & $68.04 \pm 6.05$ & 0.005 & $68.8 I \pm 4.01$ & $66.48 \pm 3.73$ & $67.44 \pm 3.45$ & $<0.001$ & 0.392 & 0.401 & 0.457 \\
\hline VAS & $4.80 \pm 0.95$ & $2.53 \pm 1.45$ & $2.66 \pm 1.48$ & $<0.00$ I & $4.82 \pm 0.87$ & $1.39 \pm 0.89$ & $1.87 \pm 1.24$ & $<0.00$ I & 0.917 & 0.005 & 0.098 \\
\hline Waddell & $3.58 \pm 1.47$ & $2.4 I \pm 1.58$ & $2.66 \pm 1.37$ & 0.001 & $3.5 \mathrm{I} \pm 1.34$ & $1.5 \mathrm{I} \pm 1.12$ & $1.88 \pm 1.25$ & $<0.001$ & 0.869 & 0.034 & 0.044 \\
\hline McGill Tot & $29.52 \pm 4.34$ & $22.10 \pm 6.00$ & $24.0 I \pm 6.25$ & $<0.00$ I & $33.17 \pm 11.05$ & $\mid 5.11 \pm 8.24$ & $\mid 8.01 \pm 8.09$ & $<0.001$ & 0.124 & 0.001 & 0.004 \\
\hline MAIA & $23.3 \pm 3.87$ & $25.48 \pm 4.31$ & $24.96 \pm 3.86$ & $<0.00$ I & $22.59 \pm 4.13$ & $27.78 \pm 3.39$ & $26.76 \pm 3.68$ & $<0.001$ & 0.546 & 0.024 & 0.097 \\
\hline SF-12 PCS & $40.88 \pm 7.85$ & $44.10 \pm 8.14$ & $42.30 \pm 9.31$ & 0.011 & $40.95 \pm 5.77$ & $47.40 \pm 5.19$ & $44.91 \pm 7.85$ & $<0.001$ & 0.858 & 0.141 & 0.308 \\
\hline SF-12 MCS & $44.73 \pm 8.92$ & $47.15 \pm 8.55$ & $46.89 \pm 8.09$ & 0.033 & $43.32 \pm 7.45$ & $48.42 \pm 4.73$ & $47.93 \pm 6.47$ & $<0.001$ & 0.417 & 0.895 & 0.985 \\
\hline
\end{tabular}

Notes: Within-group analyses were performed using Friedman's analysis for the two groups at the 3 evaluation times. Between-group statistical analyses were reported in the last 3 columns and refer to $p$-values of Mann-Whitney $U$ test between (TMG versus SMG) groups for pain (VAS; Waddel Index, McGill Tot), quality of life (SF-I2 PCS and SF-12 MCS), interoceptive awareness (MAIA Tot), HRV and coherence ratio. Data shown as mean \pm standard deviation. Statistical significance is shown in bold ( $p<0.05$ or $p<0.025)$.

Abbreviations: TMG, traditional massage therapy group; SMG, experimental massage therapy group; HRV, heart rate variability; VAS, visual analog scale; Tot, total; MAIA, Multidimensional Assessment of Interoceptive Awareness Questionnaire; SF-12 PCS, Short Form - 12 health survey - physical health scale; MCS, mental health scale; T0, baseline; TI, end of the treatment program; T2, 3-month follow-up. 


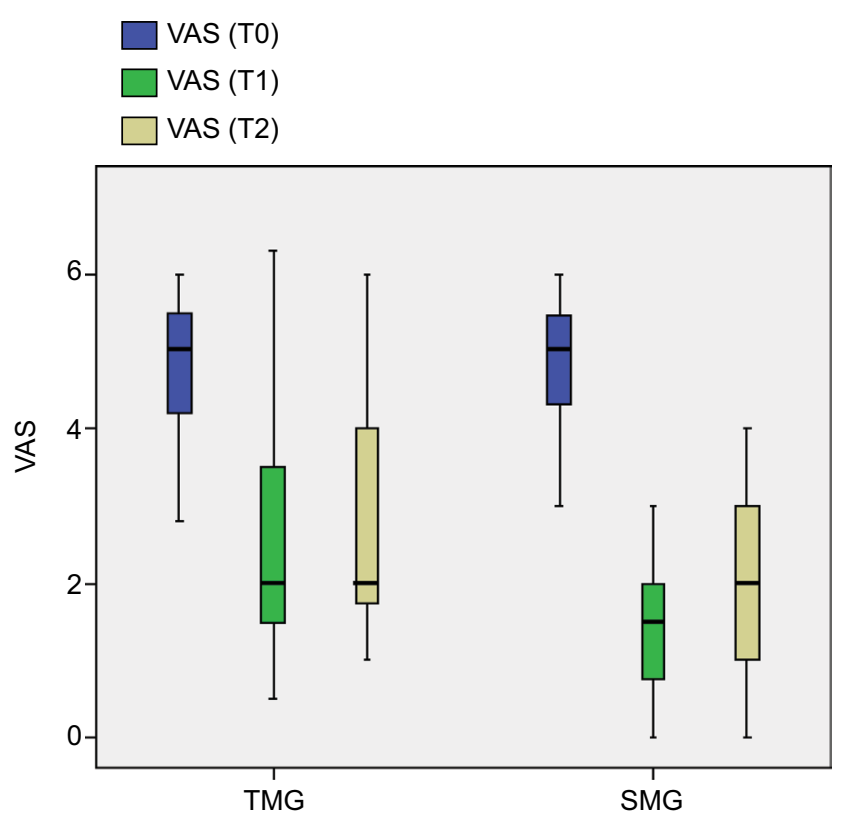

Figure $4 \mathrm{VAS}$ at TO, TI, and T2 for the two groups for Mann-Whitney $U$ test. Notes: The boxes show the lower quartile, median (middle line in box), and upper quartile values. The whiskers represent the most extreme values within 1.5 times the interquartile range from the ends of the box.

Abbreviations: VAS, visual analog scale; TMG, traditional massage therapy group; SMG, experimental massage therapy group; T0, baseline; TI, end of the treatment program; T2, 3-month follow-up.

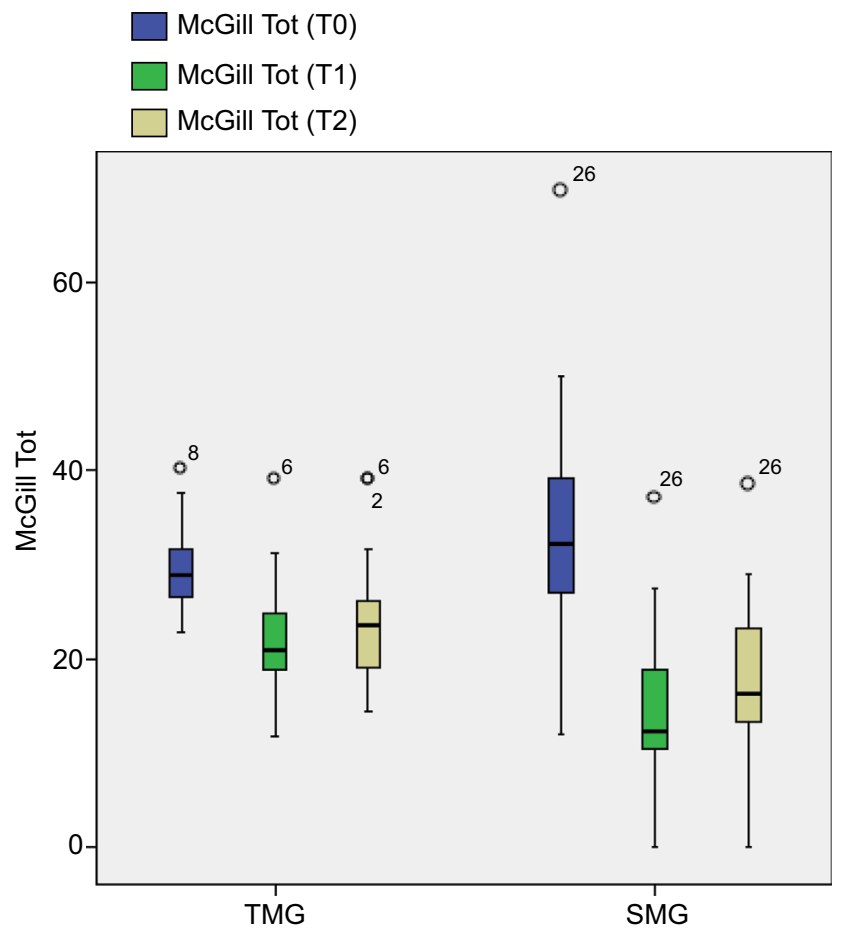

Figure 5 McGill Pain Questionnaire (McGill Tot) at T0, TI, and T2 for the two groups for Mann-Whitney $U$ test.

Notes: The boxes show the lower quartile, median (middle line in box), and upper quartile values. The whiskers represent the most extreme values within 1.5 times the interquartile range from the ends of the box and the circles represent data with values beyond the ends of whiskers.

Abbreviations: TMG, traditional massage therapy group; SMG, experimental massage therapy group; Tot, total; T0, baseline; TI, end of the treatment program; T2, 3-month follow-up.
Considering that the minimal clinically important difference in pain on the $\mathrm{VAS}^{36}$ should be at least $1.5 \mathrm{~cm}$ for patients with subacute or CLBP, the SMG met this goal, in contrast to the TMG, as shown in Table 2.

Moreover, if we consider the body as a receptive surface, touch through massage can help rebuild inconsistent information between the algic region and CNS to overcome the somesthetic and kinesthetic inconsistency in chronic pain. ${ }^{37}$ Other research has confirmed that increasing tactile and somatosensory stimuli during rehabilitation for CLBP reduces pain. ${ }^{38}$ In CLBP, tactile processing is disrupted as a neglect-like syndrome; ${ }^{39,40}$ thus, the request by the PT to the patient to pay attention to how he perceives the massage in areas that are free of back pain and then move these pleasurable sensations to the painful area helps restore adequate tactile sensory perception and recognition for reintegration of total body perception. ${ }^{41}$

Further, with regard to how chronic pain implies a decrease in parasympathetic activation, ${ }^{42,43}$ we noted a correlation between VAS score and $\mathrm{CR}$, indicating that most patients relax in the hands of a PT and that the intensity of CLBP decreases to a greater extent, likely through better activation of the vagal system and a rebalancing between the sympathetic and parasympathetic systems ( $>$ CR in the SMG).

Also, IA changed for MAIA AR and N scores in the SMG versus TMG at T1: AR - the ability to sustain attention and control body sensation - and $\mathrm{N}$ - the awareness of uncomfortable, comfortable, and neutral body sensations - are important indices of the efficacy of the new massage approach.

As the body-self neuromatrix theory of pain states, the perception of painful stimuli does not result from the brain's passive registration of tissue trauma but from its active generation of subjective experiences through a network of neurons, known as the neuromatrix. ${ }^{44}$ For rehabilitation, is important to consider that the neuromatrix, the primary mechanism that generates the neural pattern that produces pain, is genetically determined but modified by sensory experience. In our study, this experience was represented by the preparatory phase that was pleasant to the touch, performed before every session of the traditional massage treatment to direct attention of the patient away from pain. Also, the body-self neuromatrix theory subserves major psychological dimensions that correlate with the IA.

With regard to the MPQ, the SMG experienced a significant reduction in the affective component, which is linked to tension, fear, and the autonomic characteristics of pain.

The SMG showed significant improvement in perceived quality of life on the SF-12 scale for physical and mental 
items, whereas the TMG did not experience any notable changes during treatment versus baseline. Patients in the SMG appeared to change their perception of pain more extensively, thereby improving their quality of life, even with intensive treatment protocols for shorter times.

One of the limitations of this study was that we did not use scales to assess mood or psychological profile at baseline. Also, it lacked a longer follow-up ( $>6$ months) and a placebo group, preventing us from determining the true relative efficacy of the 2 rehabilitation approaches. Moreover, we cannot exclude the possibility of an attention bias that was related to the number of participants in the SMG versus the traditional approach.

\section{Conclusion}

A new massage technique with a preparatory phase that is pleasant to the touch directs the attention of the patient away from pain, with greater effects compared with the traditional treatment, consistent with the new theoretical framework for CLBP.

\section{Abbreviations}

ANS, autonomic nervous system; AR, attention regulation; $\mathrm{BL}$, body listening; BP, bodily pain; BMI, body mass index; CLBP, chronic low back pain; CNS, central nervous system; CR, coherence ratio; EA, emotional awareness; GH, general health; HRV, heart rate variability; IA, interoceptive awareness; LBP, low back pain; MAIA, Multidimensional Assessment of Interoceptive Awareness Questionnaire; MCS, mental health scale; MH, mental health; min, minutes; MPQ, McGill Pain Questionnaire; N, noticing; ND, not distracting; NSAIDs, nonsteroidal anti-inflammatory drugs; NW, not worrying; NWC, number of words chosen; PCS, physical health scale; PPI, present pain intensity; PR, physical role; PRIA, pain rating index affective subscale; PRIE, pain rating index evaluative subscale; PRIS, pain rating index sensory subscale; PT, physiotherapist; RE, emotional role; RMB, Rosen Method Bodywork; S/A, report between sensory and affective dimension; SF-12, Short Form - 12 Health Survey; SF, social function; SIMFER, Italian Society of Physical and Rehabilitation Medicine; SMG, experimental massage therapy group; SR, self-regulation; T, trusting; TMG, traditional massage therapy group; VAS, visual analog scale; VT, vitality.

\section{Author contributions}

TP and MCV conceived the study, participated in its design and coordination, coordinated the training staff, and drafted the manuscript. GP participated in the protocol development. FZ and CT participated in the design and coordination of the study and GM collected the data. MI provided the statistical analysis and interpretation of the data. All authors contributed toward data analysis, drafting, and revising the manuscript and gave final approval of the version to be published.

\section{Disclosure}

The authors report no conflicts of interest in this work.

\section{References}

1. Hoy D, Bain C, Williams G, et al. A systematic review of the global prevalence of low back pain. Arthritis Rheum. 2012;64(6):2028-2037.

2. van Middelkoop M, Rubinstein SM, Kuijpers T, et al. A systematic review on the effectiveness of physical and rehabilitation interventions for chronic non-specific low back pain. Eur Spine J. 2011;20(1):19-39.

3. Furlan $A D$, Imamura M, Dryden T, Irvin E. Massage for low back pain: an updated systematic review within the framework of the Cochrane Back Review Group. Spine (Phila Pa 1976). 2009;34(16):1669-1684.

4. Brosseau L, Wells GA, Poitras S, et al. Ottawa Panel evidence-based clinical practice guidelines on therapeutic massage for low back pain. J Bodyw Mov Ther. 2012;16(4):424-455.

5. Bervoets DC, Luijsterburg PA, Alessie JJ, Buijs MJ, Verhagen AP. Massage therapy has short-term benefits for people with common musculoskeletal disorders compared to no treatment: a systematic review. J Physiother. 2015;61(3):106-116.

6. Cherkin DC, Sherman KJ, Kahn J, et al. A comparison of the effects of 2 types of massage and usual care on chronic low back pain: a randomized, controlled trial. Ann Intern Med. 2011;155(1):1-9.

7. Frey Law LA, Evans S, Knudtson J, Nus S, Scholl K, Sluka KA. Massage reduces pain perception and hyperalgesia in experimental muscle pain: a randomized, controlled trial. J Pain. 2008;9(8):714-721.

8. Furlan AD, Giraldo M, Baskwill A, Irvin E, Imamura M. Massage for low-back pain. Cochrane Database Syst Rev. 2015;(9):CD001929.

9. Kumar S, Beaton K, Hughes T. The effectiveness of massage therapy for the treatment of nonspecific low back pain: a systematic review of systematic reviews. Int J Gen Med. 2013;6:733-741.

10. Lee HJ, Ko YL. Back massage intervention for relieving lower back pain in puerperal women: a randomized control trial study. Int $J$ Nurs Pract. 2015;21 Suppl 2:32-37.

11. Berry ME, Chapple IT, Ginsberg JP, Gleichauf KJ, Meyer JA, Nagpal ML. Non-pharmacological intervention for chronic pain in veterans: a pilot study of heart rate variability biofeedback. Glob Adv Health Med. 2014;3(2):28-33.

12. Pal GK, Ganesh V, Karthik S, Nanda N, Pal P. The effects of short-term relaxation therapy on indices of heart rate variability and blood pressure in young adults. Am J Health Promot. 2014;29(1):23-28.

13. Cellini N, Mioni G, Levorato I, Grondin S, Stablum F, Sarlo M. Heart rate variability helps tracking time more accurately. Brain Cogn. 2015;101: $57-63$.

14. Markil N, Whitehurst M, Jacobs PL, Zoeller RF. Yoga Nidra relaxation increases heart rate variability and is unaffected by a prior bout of Hatha yoga. J Altern Complement Med. 2012;18(10):953-958.

15. Sripongngam T, Eungpinichpong W, Sirivongs D, et al. Immediate effects of traditional thai massage on psychological stress as indicated by salivary alpha-amylase levels in healthy persons. Med Sci Monit Basic Res. 2015;21:216-221.

16. Craig AD. How do you feel? Interoception: the sense of the physiological condition of the body. Nat Rev Neurosci. 2002;3(8):655-666.

17. Haase L, Stewart JL, Youssef B, et al. When the brain does not adequately feel the body: links between low resilience and interoception. Biol Psychol. 2016;113:37-45.

18. Labrenz F, Icenhour A, Benson S, Elsenbruch S. Contingency awareness shapes acquisition and extinction of emotional responses in a conditioning model of pain-related fear. Front Behav Neurosci. 2015;9:318.

19. Zeidan F, Martucci KT, Kraft RA, Gordon NS, McHaffie JG, Coghill RC. Brain mechanisms supporting the modulation of pain by mindfulness meditation. J Neurosci. 2011;31(14):5540-5548. 
20. Duschek S, Montoro CI, Reyes Del Paso GA. Diminished interoceptive awareness in fibromyalgia syndrome. Behav Med. Epub 2015 Oct 2.

21. Tsay A, Allen TJ, Proske U, Giummarra MJ. Sensing the body in chronic pain: a review of psychophysical studies implicating altered body representation. Neurosci Biobehav Rev. 2015;52:221-232.

22. Lithwick A, Lev S, Binshtok AM. Chronic pain-related remodeling of cerebral cortex - 'pain memory': a possible target for treatment of chronic pain. Pain Manag. 2013;3(1):35-45.

23. Fairhurst M, Fairhurst K, Berna C, Tracey I. An fMRI study exploring the overlap and differences between neural representations of physical and recalled pain. PLoS One. 2012;7(10):e48711.

24. Chan SC, Chan CC, Kwan AS, Ting KH, Chui TY. Orienting attention modulates pain perception: an ERP study. PLoS One. 2012;7(6):e40215.

25. Fogel A. Better or worse: a study of day-to-day changes over five months of rosen method bodywork treatment for chronic low back pain. Int $J$ Ther Massage Bodywork. 2013;6(3):14-24.

26. Huskisson EC. Measurement of pain. J Rheumatol. 1982;9(5):768-769.

27. Melzack R. The McGill Pain Questionnaire: major properties and scoring methods. Pain. 1975;1(3):277-299.

28. Waddell G, Main CJ. Assessment of severity in low-back disorders Spine (Phila Pa 1976). 1984;9(2):204-208.

29. Mehling WE, Price C, Daubenmier JJ, Acree M, Bartmess E, Stewart A The Multidimensional Assessment of Interoceptive Awareness (MAIA). PLoS One. 2012;7(11):e48230.

30. Gandek B, Ware JE, Aaronson NK, et al. Cross-validation of item selection and scoring for the SF-12 Health Survey in nine countries: results from the IQOLA Project. International Quality of Life Assessment. J Clin Epidemiol. 1998;51(11):1171-1178.

31. Ware J Jr, Kosinski M, Keller SD. A 12-Item Short-Form Health Survey: construction of scales and preliminary tests of reliability and validity. Med Care. 1996;34(3):220-233.

32. McCraty R, Shaffer F. Heart rate variability: new perspectives on physiological mechanisms, assessment of self-regulatory capacity, and health risk. Glob Adv Health Med. 2015;4(1):46-61.
33. Selvaraj N, Jaryal A, Santhosh J, Deepak KK, Anand S. Assessment of heart rate variability derived from finger-tip photoplethysmography as compared to electrocardiography. J Med Eng Technol. 2008;32(6): 479-484.

34. James HC, David MP. Fondamenti clinici della massoterapia. Anatomia integrata e trattamenti [Clinical fundamentals of massage therapy. Integrated and treatments anatomy]. Piccin. 2009:1-150. Italian.

35. Ostelo RW, de Vet HC. Clinically important outcomes in low back pain. Best Pract Res Clin Rheumatol. 2005;19(4):593-607.

36. Dworkin RH, Turk DC, Wyrwich KW, et al. Interpreting the clinical importance of treatment outcomes in chronic pain clinical trials: IMMPACT recommendations. J Pain. 2008;9(2):105-121.

37. Boesch E, Bellan V, Moseley GL, Stanton TR. The effect of bodily illusions on clinical pain: a systematic review and meta-analysis. Pain. 2016 157(3):516-529.

38. Moseley GL, Gallagher L, Gallace A. Neglect-like tactile dysfunction in chronic back pain. Neurology. 2012;79(4):327-332.

39. Morone G, Iosa M, Paolucci T, et al. Efficacy of perceptive rehabilitation in the treatment of chronic nonspecific low back pain through a new tool: a randomized clinical study. Clin Rehabil. 2012;26(4):339-350.

40. Birklein F, Maihöfner C. Neglect your back to control your pain? Neurology. 2012;79(4):300-301.

41. Leifert-Fiebach G, Welfringer A, Babinsky R, Brandt T. Motor imagery training in patients with chronic neglect: a pilot study. NeuroRehabilitation. 2013;32(1):43-58.

42. Tracy LM, Ioannou L, Baker KS, Gibson SJ, Georgiou-Karistianis N, Giummarra MJ. Meta-analytic evidence for decreased heart rate variability in chronic pain implicating parasympathetic nervous system dysregulation. Pain. 2016;157(1):7-29.

43. Daffada PJ, Walsh N, McCabe CS, Palmer S. The impact of cortical remapping interventions on pain and disability in chronic low back pain: a systematic review. Physiotherapy. 2015;101(1):25-33.

44. Melzack R. Pain and the neuromatrix in the brain. J Dent Educ. 2001; 65(12):1378-1382.
Journal of Pain Research

\section{Publish your work in this journal}

The Journal of Pain Research is an international, peer reviewed, open access, online journal that welcomes laboratory and clinical findings in the fields of pain research and the prevention and management of pain. Original research, reviews, symposium reports, hypothesis formation and commentaries are all considered for publication

\section{Dovepress}

The manuscript management system is completely online and includes a very quick and fair peer-review system, which is all easy to use. Visit http://www.dovepress.com/testimonials.php to read real quotes from published authors. 